\title{
22
}

\section{An Approach to TTCN-Based Test Execution}

\author{
Yamin Wang, Jianping Wu and Ruibing Hao \\ Department of Computer Science \\ Tsinghua University, Beijing 100084, China
}

\begin{abstract}
Test execution plays an important role in the protocol conformance testing system. We can classify existing test execution methods into two types: Compiling-based Test Execution and Interpreting-based Test Execution. This paper gives an approach to test execution based on a formal test suite specification given in TTCN. In our protocol conformance testing system, the test suites in TTCN are executed directly by parallel interpreting according to the operational semantics of TTCN. This method is protocol independent and can obtain more efficient test execution than other methods. Moreover, this paper also presents an overview of our protocol conformance testing system and the structure design of the TTCN-based Test Execution implementation.
\end{abstract}

Keyword Codes: C.2.2; D.2.2; F.4.3

Keywords: Network Protocol; Testing and Debugging; Formal Languages

\section{Introduction}

Protocol Conformance Testing System (PCTS) is the most principal facility to carry out protocol conformance testing. Test Execution (TE) is an engine in all the components of a PCTS. It interacts with all other components of a PCTS, and controls testing process according to the content of Test Suite (TS) and simultaneously generate all information required to produce Test Report (TR)

The most existing TEs do not use formal methods, and have quite different test execution processes. They usually define a particular internal notation to describe the test suite to be executed. There are two kinds of methods to execute the test suites. One is that the test suite are compiled into an executable machine codes, and then the machine codes run step by step. Another is that the test suite are interpreted to execute. The first one will take long time for the compiling, and is hard to maintain these executable machine codes. The second one will be inefficient because the interpreting and executing of the TS is a sequential process. Both these methods are less flexible and particular protocol or system circumstance dependent.

In recent years, ISO have gradually developed the test suites for their standard protocols, and these test suites are described in TTCN. We put forward a method of TTCN-based TE. This method organizes its testing process on the basis of the TS in TTCN, and uses parallel interpreting to raise test efficiency. Some characteristics of this method are in the following:

- Accordance with ISO protocol testing standards. All the ideas, methods and terminology's adopted in our PCTS strictly follow ISO 9646 protocol testing standard. In our PCTS, all the protocol reference implementations and the services accord with corresponding ISO standards. TS is formally described in TTCN, which is proposed by ISO 9646-3[13].

- Flexibility and independence. In our TTCN-based TE, test suites is executed according to the operational semantics of TTCN, so this method is flexible and independent on 
the protocol being tested. It can be seen as only an execution mechanism for any TS in TTCN. So it can test all the protocols whose TS in TTCN. Any protocol appeared newly can be accepted by our PCTS, only with fulfillment of its TS in TTCN.

- Efficient test execution. When a test case is executed, the most possible next test case is being interpreted. The parallel interpreting allows PCTS to improve its executing efficiency. TTCN-based TE interprets a test case just before its execution to provide testing operator with the more possibilities to control the testing process, such as Single Step Testing and Supervising.

This paper is structured as follows. In section 2, we summary the existing methods of test execution, and classify them into two types. Section 3 gives our TTCN-based TE method. Section 4 deals with an overview of our PCTS, the structure design of TTCN-based TE implementation, and some technical details. Test execution of X.25 LAPB using TTCN-based TE method is discussed. Finally, we give a conclusion.

\section{Test Execution}

Correct and precise description of TS is the premise of successful development of a PCTS, because TE, the engine of a PCTS, regards TS as its operating object like the instructions in a computer. The style of a TS will determine the style of a TE, and further that of a PCTS. We can classify the existing TE methods into two types:

- Compiling-based Test Execution (CTE);

- Interpreting-based Test Execution (ITE)

Using ISO terminology's, those test suites in internal notation or in the TTCN can be called as an Abstract Test Suites (ATS), and those executable machine codes can be called an Executable Test Suites (ETS).

\subsection{Compiling-Based Test Execution}

The CTE is composed of three function components: translator, compiler and executor They are called according to the test execution control information from PCTS. Translator converts ATS into a computing languages, such as C or PASCAL. The test suites in the computing language is compiled to generate ETS. It is worthy of note that both the translator and compiler will take an entire test suite of a protocol as an object they handle, while the executor only run executable test case one by one. The functions of an executor include receiving the test execution control information and making concerned executable test case to run. It does not go deep into every concrete execution step, such as decoding and encoding etc.

The CTE may have a comparatively efficient testing, but the maintenance and management of the ETS are very troublesome so that it is hard to do some artificial interference for the purpose of supervision and observation.

To apply for massive TS and successive testing, many famous PCTS's adopt this kind of TE to perform efficiently. For example, IDACOM PCTS and XRTLE PCTS. In IDACOM PCTS, the TTCN Translator is a workstation-based tool to generate $\mathrm{C}$ code corresponding to an ATS specified in the Machine Processable(MP) form of TTCN, and the $\mathrm{C}$ code can be compiled using any $\mathrm{C}$ compiler [20]. The XRTLE PCTS of USA Alctel TITN Inc. comprises the interpreter of the object code produced by the compilation of programs written in XSDIL [26]. In fact, this interpreter also does those function the executor does as mentioned above.

\subsection{Interpreting-Based Test Execution}

In the interpreting-based test execution, the TE completes the derivation from ATS to the ETS while organizing testing. This feature may make the TE lower efficient. But the PCTS with this kind of test execution provides users many more chances to dynamically control and 
monitor the testing process. Compared with CTE, the ITE has a different test execution process. In the CTE, the executor only calls each executable test case by its file name and then run the file. In the ITE, the interpreter and the executor work together to interpret and run those abstract test case one by one. Those executable test cases only exist during test execution.

In a PCTS named COAST[15], which adopted ITE, the test interpreter (TPS/I) is the component of the real tester. The interpreter is completely independent of any specifies of the SUT, the protocol being tested and the underlying service. It interprets the parameterized abstract test cases of the PATG and interacts with the test execution system, which processes single parameterized abstract test events (PATES).

In the UBC Protocol Testing Environment, when a test execution command (together with a test case identifier) is received, the test manager loads the test case from the ETS (Executable Test Suite) behavior library and invokes the test suite processor to execute it. Here the test cases in the ETS behavior library are an intermediate form between the abstract test suite and the executable test case. The test case can be interpreted and executed in sequence by the test suite processor.

\subsection{Comparison between the two test executions}

It is clear that the CTE and ITE have its virtues and weakness respectively. During the testing process, the relatively higher efficiency of the CTE is obvious. While the ITE has a lower testing execution speed, because of its excessive steps, such as reading test events, encoding/decoding and forming test logs, etc.

Concerned with the modifiability, for the ITE any revision of the TS will not cause the reconstruction of the $\mathrm{TS}$, because every test parameter comes into effect only during test execution. But for the CTE, it is different. If the revision is limited in a certain test case, it is easy to handle, with only recompilation of this test case. If the revision involves the parameters or PDUs, it is bound to recompile all the TS, which obviously costs too much.

The ITE is liable to set up view point of testing process and provide such function as single step test. These advantage attributes to that each test event is under the control of executor. This characteristics meets the needs of the flexibility and independent of PCTS. But for the CTE, the achievement of this function will cause the augment of control tributary, which leads to large scale compiled test cases.

\section{TTCN-based Test Execution}

ISO is gradually developed the test suites for their standard protocols, and these test suites are described in TTCN, which is defined by ISO 9646-3[13]. This trend demands the application of the TTCN-based TE. Both of the CTE and the ITE, as mentioned above, should be improved to meet the needs of the flexibility, independence and efficiency of the PCTS's. We put forward a method of TTCN-based TE here. This method organizes its testing process on the basis of the TS in TTCN, and uses a parallel interpreting to raise test efficiency.

\subsection{Adoption of TTCN}

The flexibility and independence of a PCTS demands that the TSs of various protocols are described in a same formal description language, while TE is the only handler to this kind of TS. The modifiability of TS and PCTS itself demands that TE is independent of the selection and management of TS. That means that the revisions to a TS almost make no effect on the design of TE.

The TTCN developed by ISO uses a Tree and Tabular Combined Notation to describe TS. As the definition of TTCN in [13], TTCN provides a notation which is independent of test methods and protocols. Also, TTCN has a clearly defined semantics. The definition of its semantics follows a two-phases approach. In the first phase concrete TTCN texts are mapped 
to a simplified structure, that can be manipulated during the second phases. The second phase can be regarded as the description of a TTCN machine, that interprets these simplified TTCN texts. In summary, normalized forms and clearly defined operational semantics make it possible to be generated and distinguished automatically by machine.

\subsection{Parallel Interpreting}

From the structure of test cases in TTCN, we find out their tree relation between one test case and its following test cases. After one test case is executed, there will be only one of possible test cases to be executed. This tree structure of test cases allows us to arrange some interpreting of the most possible next test case while one test case is executed. This parallel interpreting of test cases is very like the parallel processing of computer instructions. The interpreting will have enough time to be finished, because the test execution of one test case is often composed of sending a PDU, waiting a remote response, making a verdict and reporting testing result to $\log$ file etc. These works will take many time definitely.

In the TTCN-based TE, the test execution of one test case is divided two periods: interpreting and execution. During any time of testing process, the test execution of the current test case and the interpretation of the most possible next test case are paralleled. Here the executable test case is derived by the Interpreter and acted by the Test Execution. All these efforts will make remarkable improvement in efficiency. This improvement in efficiency will be very worth for the protocol testing of high speed computer networks, such as ATM etc.

\section{Design of TTCN-Based TE}

\subsection{Overview of our PCTS}

An integrated OSI protocol conformance testing system has been developed at Tsinghua University. Since the beginning, the OSI PCTS has aimed to provide a basic platform for developing protocol conformance testing, and at the same time provide real test system for testing the main OSI protocols. The following objectives guided our design and implementation effort:

- Accordance with ISO protocol testing standards: The adoption of OSI protocol testing methodology will make this system standard and practical, as well as comparable and sharable.

- Use of formal methods: The standard formal methods, such as TTCN and ASN.1 should be used in the system for a sound theoretical foundation.

- Ease of adaptation: For the more adaptability, the system should be designed, as much as possible, to be protocol independent, test method independent and test configuration independent.

- TTCN-based test execution: The test execution of the system is organized on the basis of interpreting the test case according to the semantics of TTCN in parallel, for the purpose of more efficient and independent test execution than any existing methods.

- $X$ window-based test presentation and user interface: The $X$ window-based graphic user interface should be used for a multiple window test presentations and a very friendly user interaction. This standard user interface will make many tools included in the system also usual in the other applications, e.g. the X window-based TTCN editor;

- Object-oriented test software environment: The test software environment should provide a methodology of test software organization and programming. The object-oriented test software environment will make system modules conceptualized and reused.

The different system components in our PCTS as well as their interrelations are shown in Figure 1. The PCTS is composed of the following main components: Test Presentation and User Interface (TPUI), Test Case Selection and Management (TCSM), Test Execution (TE), 
Test Result Report and Analysis (TRRA), Service Provider (SP), Formal Support Tools (FST), and Test Software Environment (TSE).

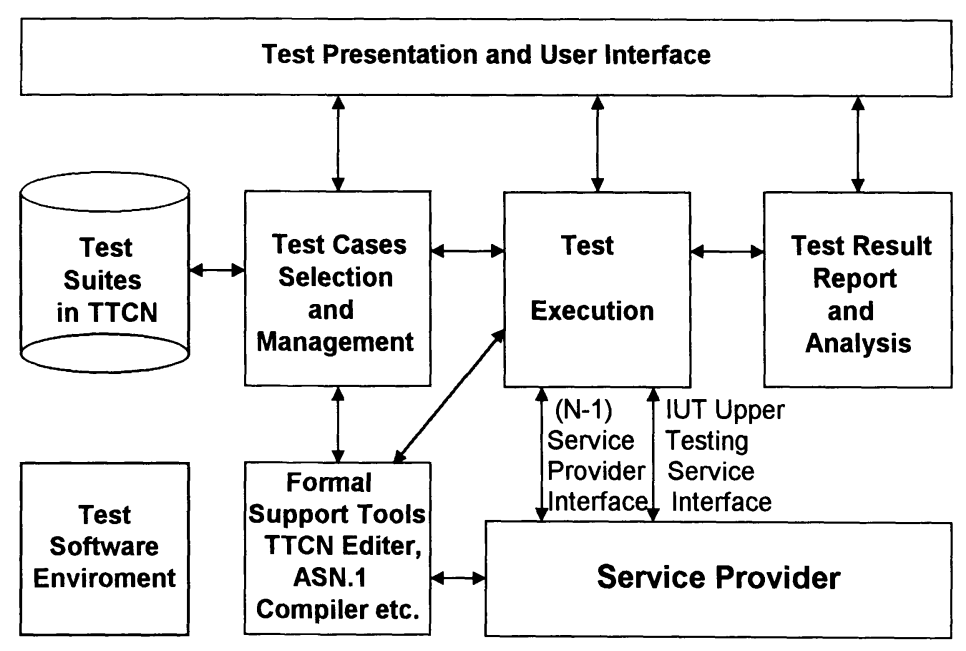

Figure 1. Architecture of Protocol Conformance Testing System

\subsection{Structure Design of TTCN-Based TE}

The Test Execution implements the principle of the TTCN-based TE mentioned above. Figure 2. shows its internal structure and interactions with other system components. The function of test execution is finished by two parallel processes: Interpreter and Test Executor. The two processes co-operate each other to execute these test cases. Share Memory is an intermediate storage for the parameterized and executable test case which will is the most possible next test case to be executed. Message Send/Receive Queues are used for the communication with the IUT to be tested. Some details of the structure and design are as follows

\section{Interpreter}

The interpreter is a process which finishes, as parallel as possible, the interpreting from an abstract test case to an executable test case. It accepts a request of getting an executable test case from the test executor, and forward it to the TCSM, When an identifier of an corresponding abstract test case arrives from the TCSM, it is need to check if this abstract test case has been interpreted in advance and is stored in the Share Memory. If so, this executable test case is sent to the Test Executor to be executed. If the Share Memory is empty or the obtained abstract test case is not corresponding with an executable test case storied in the Share Memory, the Interpreter must interpret the obtained abstract test case into an executable test case, and send it to the Test Executor. No matter which condition above happens, the Interpreter will request the TCSM to select the most possible next test case to be executed, interpret and story it in the Share Memory. It is worthy of note that the dynamic selection of the test case is performed by the TCSM, based on a selection strategy, and that the next possible test case is interpreted while a test case is being executed. After the interpreting, the Interpreter waits for the test results, and sends it to the TCSM. 
The procedure of interpretation can be decomposed into three steps:

Syntax definition: Check the syntax of a TTCN-based test case;

Static semantics definition: Check the static semantics of test case;

Transformation to an evaluation tree: Construct an evaluation tree from a concrete TTCN text that is syntactically and static semantically correct. The transformation algorithm can be divided into three substeps:

- Appending of Default Behaviors;

- Removal of REPEAT Constructs;

- Expansion of Attached Trees.

After an evaluation tree has been constructed, it is stored in the Share Memory and passed to Test Execution according to its request.

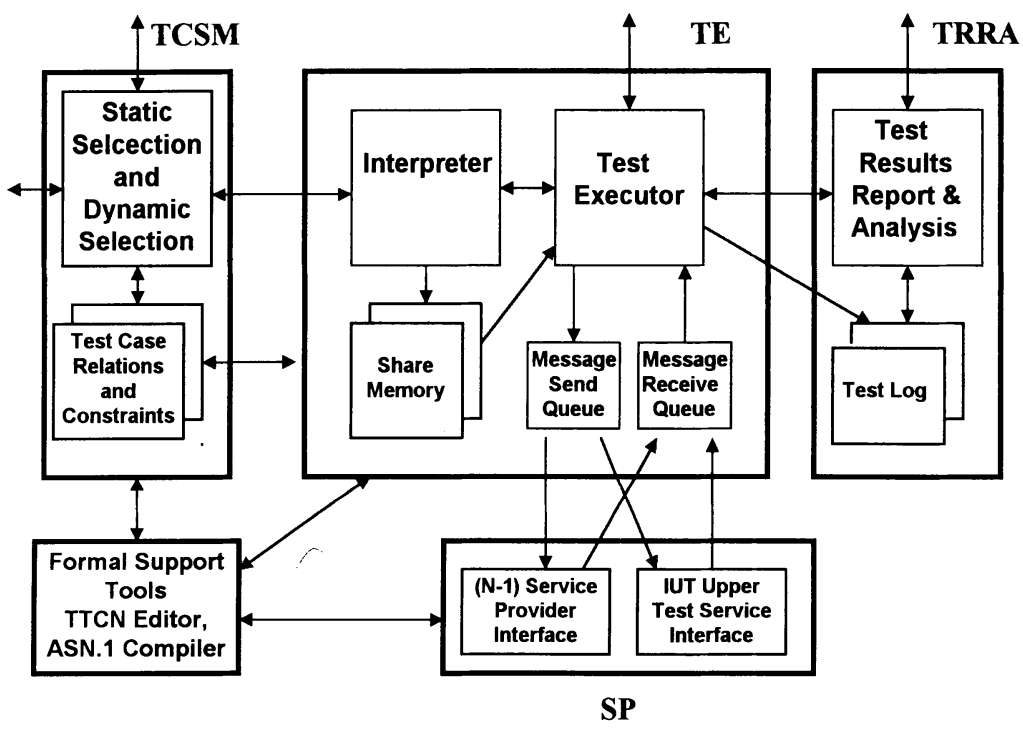

Figure 2. Structure of Test Execution

\section{Test Executor}

The Test Executor organizes test activities only based on the commands from user interface. When it accepts a command which requests the TE to begin test, it sends a request to the Interpreter for getting an executable test case which has been interpreted. After getting a respond from the Interpreter, it will execute this test case by traversing a path in the evaluation tree of this test case, and sending/receiving ASP or PDU to/from the IUT. At the same time, it also send each test even to the test log file in TRRA. When a test is finished, a test result will be produced and sent to the Interpreter. In fact, the Interpreter will forward the test results to the TCSM as an important reference to decide the most possible next test case to be executed.

The main part of Test Executor can be regarded as an abstract TTCN machine. It interprets an evaluation tree strictly according to the operational semantics of TTCN. 


\subsection{An Example of TTCN-Based TE}

The X.25 LAPB protocol has been used as an example for the evaluation of our TTCNbased TE. X.25 LAPB is data link layer protocol with exhaustive uses, and ISO and CCITT have developed a complete X.25 test suite in TTCN.

Our PCTS accepts X.25 LAPB test suite specified in TTCN.GR, and translates it into TTCN.MP. Before test execution, these test cases are analyzed to generate three static information: a test structure table, a parameter table and a test purpose table. These tables are stored and managed by SYBASE database management system, while the test cases in TTCN.MP are divided and stored into many relatively independent files which each is a test case. The above works are finished automatically and human intervention is minimized. We find out that in the testing of X.25 LAPB protocol, interpreting of the most possible next test case is much fast than the test execution of the current test case. At present, a dynamic selection strategy named "right path first" is used for selecting the most possible next test case. It is based on the suppose, that is for the most part a protocol implementation will be conformance with its protocol standard.

\section{Conclusions}

We have presented an approach to TTCN-based Test Execution. This method interprets and executes the selected and parameterized abstract test suite specified in TTCN. For the purpose in efficient, the most possible next test case is interpreted in advance while a test case is being executed. This method provides a more efficient test execution, as well as more flexibility and independent. This method takes the test cases in formal description notation TTCN as an object of test execution. This will make our protocol testing system protocol independent, abstract test method independent and test selection independent. The parallel interpreting raising test efficiency will be used for testing these existing OSI protocols, as well as for both multi-party and interoperablility testing.

Our PCTS with the TTCN-based Test Execution have been used for testing real protocols such as X.25 LAPB, X.25 PLP, and TP protocol. Current research work based on the test execution method includes evaluation of the test efficiency with other test execution methods in the fixed test quantity, and application for testing high speed network protocol such as ATM

\section{Reference}

[1] A.H.Wiles, Experiences of Computer Aided Derivation of Executable Test Suites fromAbstract Test Suites, Protocol Test Systems III, pp 185-198, 1990.

[2] Behcet Sarikaya, Conformance Testing: Architectures and Test Sequences, North-Holland, Computer Networks and ISDN Systems, Vol. 17, pp111-126, 1989.

[3] Deepinder Sidhu, Experience with Formal Methods in Protocol Development, FORTE' 89, p579-595.

[4] Deepinder Sidhu, Thomas P. Blumer, Semi-automatic Implementation of OSI Protocols, Computer Networks and ISDN Systems, Vol.18 (1989/90), pp221-238.

[5] D.Rayner, OSI Conformance Testing, North-Holland, Computer Networks and ISDN Systems, Vol.14 (1987), pp 79-98.

[6] George A. Harvey, The Routing Certification System, Protocol Specification, Testing and Verification III, pp 465-477, 1983.

[7] G.v.Bochmann, D.Desbiens, M.Dubuc, D.Ouimet and F.Saba, Test Result Analysis and Validation of Test Verdicts, Protocol Test Systems III, 1990. 
[8] Gregor V.Bochmann, Usage of Protocol Development Tools: The Results of a Survey, Protocol Specification, Testing and Verification VII, pp139-159, 1987.

[9] ISO/IEC 8882-2, X.25 DTE Conformance Testing, Part 2: Data Link Layer Conformance Test Suite, 1991.

[10] ISO/IEC 8882-3, X.25 DTE Conformance Testing, Part 3: Packet Level Conformance Test Suite, 1991.

[11] ISO IS/9646-1, OSI Conformance Testing Methodology and Framework, Part 1: General Principles, 1991.

[12] ISO IS/9646-2, OSI Conformance Testing Methodology and Framework, Part 2: Abstract Test Suite Specification, 1991.

[13] ISO IS/9646-3, OSI Conformance Testing Methodology and Framework, Part 3: The Tree and Tabular Combined Notation (TTCN), 1991.

[14] ISO IS/9646-5, OSI Conformance Testing Methodology and Framework, Part 5: Requirements on Test Laboratories and Clients for the Conformance Assessment Process, 1991.

[15] J.de Meer, J.Burmeister, A.Rennoch, V.Heymer, An Approach to a Conformance Testing Methodology and the COAST Test System, Protocol Test Systems III, pp245-262, 1990.

[16] J.P.Ansart, V.Chari, D.Simon, From Formal Description to Automated Implementation Using PDIL, Protocol Specification, Testing and Verification III, pp381-390, 1983.

[17] J.P. Favreau, R.J. Linn, S. Noghtingale, A Formal Multi-Layer Test Metrology and its Application to OSI, FORTE' 89, pp509-523, 1989.

[18] Kotaro Katsuyama and Fumiaki Sato, Strategic Testing Environment with Formal Description Techniques, IEEE Transactions on Computer, Vol. 40, No. 4, April 1991.

[19] M.McAllister, S.T. Vuong and J. Alilovic-Curgus, Automated Test Case Selection Based on Test Coverage Metrics, Computer Science Department, University of British Columbia.

[20] S.T. Chanson etc., The UBC Protocol Testing Environment, Protocol Test System VI, pp219-234, 1993.

[21] R.L. Probert, O. Monkewich, TTCN: the international notation for specifying tests of communications systems, North-Holland, Computer Networks and ISDN Systems Vol. 23 (1992), pp 417-438

[22] R.J. Linn, Conformance Testing for OSI Protocols, North-Holland, Computer Networks and ISDN Systems Vol.18 (1989/1990), pp 203-219.

[23] R.J. Linn, J.S. Nightingale, Some Experience with Testing Tools for OSI Protocol Implementations, Protocol Specification, Testing and Verification III, pp521-531, 1983.

[24] Koster, A Protocol Software Implementation and Test Environment, Protocol Test Systems III, pp31-43, 1990.

[25] Ruud Berkhout and Marc F. Witteman, Application of Multi-party Conformance Test Methods to the GSM Mobile Network System, Protocol Test Systems III, pp227-243, 1990.

[26] XRTLE Range Overview, Alctel TITN Inc., 1992. 